\title{
Influence of Irradiation on Mechanical Properties of Materials
}

\author{
V.V. Krasil'nikov ${ }^{1}$ and S.E. Savotchenko ${ }^{2}$ \\ ${ }^{1}$ Belgorod State University \\ ${ }^{2}$ Belgorod Regional Institute of Postgraduate Education \\ and Professional Retraining of Specialists \\ Russia
}

\section{Introduction}

The one of actual problems of radiation material science is to reveal plastic deformation laws, hardening and fracture ones of materials under intense external action, particularly irradiation. Herein we imply different kinds of irradiation, for instance, such a $(e, \gamma)$ beam irradiation, ion or neutron irradiation and so on. Evolution of a construction material microstructure at a high temperature trial operation is substantially conditioned by free migrating defects [1]. The processes of interaction of point defects with each other, with dislocations and interface underlie all of metal radiation hardening mechanisms [2]. In the section 1, the nonlinear model of dose dependence saturation of the yield strength is proposed on the base of the vacancy and interstitial barrier interaction. Processes of mutual recombination of vacancy and interstitial barriers and formation of vacancy and interstitial clusters are taken into consideration.

A series of different radiation defects (retardation barriers of dislocations), and their sizes, and a form of their volume distribution contribute into a yield strength increment for all of sorts of irradiation. The contribution of a barrier type is determined by conditions of irradiation and tests. At the low temperature irradiation (at the test temperatures up to $0.3 \mathrm{~T}_{\mathrm{m}}$ $\mathrm{T}_{\mathrm{m}}$ is melting temperature), interstitial atoms, and vacancies, and their clusters contribute mainly into the hardening. In the section 2, evolution of radiation barrier (vacancies and interstitials) clusters is analyzed under low temperature radiation in the presence of the most important secondary effectes: recombination and formation of divacancy complexes. It is proposed a barrier hardening model in that mechanisms of mutual annihilation of the vacancy and interstitial barriers and their clusterization play a main role.

In the section 3 unlike two preceding sections where the dose dependences are considered, the phenomenological model is formulated to describe a yield strength temperature dependence of polycrystalline materials that have undergone irradiation and mechanical experiences in a wide temperature interval including structure levels of plastic deformation. In this section, a new phenomenological model is proposed to give a suitable description of yield strength temperature dependence of some of irradiated materials in a temperature interval including plastic deformation structure levels. 


\section{Mutual recombination and clusterization effect of the vacancy and interstitial barriers on radiation hardening materials}

\subsection{Formulation of the model}

In initial stages of low temperature irradiation (up to $0.3 \cdot T_{\mathrm{m}}$ where $T_{\mathrm{m}}$ is melting temperature) at small doses, inhibiting processes of initial dislocations and their sources dominate. The point defects are connect with a dislocation and form dislocation jogs and steps but interacting with each other form interstitial - vacancy - impurity clusters. As a result, energy and geometry dislocation characteristics change substantially. A phenomenological description of these mechanisms is within the usual Granato - Lukke theory.

At large irradiation doses of metals, the processes of an elastic and contact interaction of sliding dislocations with different potential barriers begin to play a main role. Besides the separated point defects in irradiated material, the dislocations are to surmount interstitial and vacancy clusters and dislocation loops, the interstitial - vacancy - impurity clusters, precipitates, voids. The dislocation can cut a barrier, can be bent by the barrier and can go round an obstacle by a dislocation climb subject to barrier intensity and a distance between barriers. These mechanisms of barrier hardening are described by the Orowan model of athermic surmounting obstacles by dislocations [3].

At the large irradiation doses, a yield strength increment can be represented by the sum of barrier contributions of different types [3]:

$$
\Delta \sigma=\sum_{i=1}^{N} \Delta \sigma_{i},
$$

where index $i$ is a barrier type, $N$ is a number of barrier types affecting the yield strength, $\Delta \sigma_{i}$ is the yield strength increment of $i^{\prime}$ barrier type.

Up to now, there is many of experimental data and numerical theoretical models are developed to describe a dependence of barrier concentration of different types on radiation hardening power and behavior pattern of point defect clusters [4]. It is known that on earlier stage at small irradiation doses (by neutron radiation up to $2 \cdot 10^{16} \mathrm{n} / \mathrm{cm}^{2}$ ), the hardening occurs due to forming the slowly increasing interstitial clusters. The vacancy clusters begin to contribute to the hardening in increasing dose.

In these stages, the yield strength increment is described with sufficient approximation of the dependence of the form

$$
\Delta \sigma=a(\Phi t)^{n},
$$

where $a$ is a parameter depending on irradiation conditions and the research material type, $\Phi$ is density of particle flux, $t$ is irradiation time, exponent $n$ changes against the material type and the irradiation condition from 0.25 to 0.75 [3].

A saturation nature of the hardening is still not clear finally. Probable causes of the yield strength increment saturation can be such as overlapping stresses field created by radiation 
defects of certain their concentration, creation round the volume defects of defect-free zones, the beginning of the dislocation channeling and surmounting obstacles processes and so on.

In the Ref [5], the model is proposed to describe the dose dependence of the copper yield strength increment where the saturation is explained by a decreasing velocity of the forming clusters with increasing irradiate dose due to interaction between the available clusters and newly forming ones.

Here the model is proposed to describe the dose dependence of the yield strength increment taking into account of vacancy and interstitial barrier interaction.

We consider that vacancies and interstitial atoms make a main contribution to the yield strength increment of a certain material at some of irradiation conditions. They are barriers to play the main role in the hardening at the low temperature irradiation. Therefore, in the proposed model $N=2$; the index values of $i=1$ and 2 correspond to the vacancies and interstitial atoms (and their clusters). Then in this model Eq (2.1) takes the form:

$$
\Delta \sigma=\Delta \sigma_{1}+\Delta \sigma_{2} .
$$

For all of the obstacle types, the metal yield strength increment conditioned by dislocation deceleration is described as [2]:

$$
\Delta \sigma_{i}=\alpha_{i} \mu b\left(C_{i} d_{i}\right)^{1 / 2}, i=1,2,
$$

where $\alpha_{i}$ is the parameters characterizing $i^{\prime}$ barrier intensity (a fixed quantity for some of barrier types, material and irradiation condition), $\mu$ is the shear modulus, $b$ the Burgers vector length, $C_{i}$ the volume density of $i^{\prime}$ barrier type, $d_{i}$ their average size. For instance, the vacancy and interstitial have the average size $\sim 10 \mathrm{~nm}$, and the parameters characterizing barrier intensity has the value about 0.2 [2].

The present model is based on the system equations for the volume densities of the radiation- induced nonequilibrium vacancies and interstitial barriers $C_{1}, C_{2}$ :

$$
\left\{\begin{array}{l}
\frac{d C_{1}}{d \tau}=K_{1}-\frac{C_{1}}{\tau_{1}}-\gamma_{12} C_{1} C_{2}-\gamma_{1} C_{1}^{2} \\
\frac{d C_{2}}{d \tau}=K_{2}-\frac{C_{2}}{\tau_{2}}-\gamma_{12} C_{1} C_{2}-\gamma_{2} C_{2}^{2} .
\end{array}\right.
$$

where $\tau=\Phi t$, $\Phi$ is density of particle flux, $t$ irradiation time, $K_{i}, i=1,2$, the intensities of forming the radiation - induced vacancy and interstitial barriers, $\gamma_{i}$ are the coefficients of barrier recombination and characterize forming the clusters of acceptable barrier type (it can be named as clusterization coefficients), $\gamma_{12}$ the coefficient of mutual recombination of the annihilating vacancy and interstitial barriers, the coefficients $\tau_{i}^{-1}$ can be represented by the form: $\tau_{i}^{-1}=K_{i} V_{i}$, where $V_{i}$ are the effective volumes of interaction of the certain barriers with each other.

The first terms of the equation system (2.5) describe the intensity of increasing the volume barrier densities of the acceptable type, the second ones correspond to decreasing the volume barrier densities due to absorbing the barriers on natural sinks: voids, dislocations, 
dislocation network, grain boundaries and so on. In the proposed model, the mechanisms of the mutual annihilation of vacancy and interstitial barriers and their clusterization are assigned. The third terms of the system (2.5) describe decreasing the barrier densities due to of the mutual annihilation of two different type barriers, and the fourth ones do due to forming the clusters of two barriers of the same type.

To find the volume densities of the radiation - induced nonequilibrium vacancies and interstitial atoms it is necessary to set up their the initial values:

$$
C_{i}(0)=C_{i}^{(0)}, i=1,2 .
$$

Thus, the mathematical formulation of the present model is the Cauchy problem for the system of nonlinear differential equations (2.5). The volume barrier densities found as a result of solution of the Cauchy problem (2.5), (2.6) determine the yield strength increment according to Eqs (2.3), (2.4).

\subsection{Dose saturation features of the yield strength subject to annihilation effects of the vacancy and interstitial barriers}

At the beginning, we consider the yield strength behavior against the material irradiation dose taking into account of only the mutual recombination of the vacancy and interstitial barriers that is their annihilation. Upon that, the effects of forming the barrier clusters of the same type are not considered. The barrier annihilation effects but no their clusterization play the main role in the radiation hardening. Therefore $\gamma_{12}>\gamma_{i}, i=1,2$, and it is possible to neglect the last terms of $\gamma_{i} C_{i}^{2}$ in every equation of the system (2.5).

In this approximation the system (2.5) takes the form:

$$
\left\{\begin{array}{l}
\frac{d C_{1}}{d \tau}=K_{1}\left(1-V_{1} C_{1}\right)-\gamma_{12} C_{1} C_{2}, \\
\frac{d C_{2}}{d \tau}=K_{2}\left(1-V_{2} C_{2}\right)-\gamma_{12} C_{1} C_{2} .
\end{array}\right.
$$

As Eqs (2.7) has not an analytic solution, we illustrate a dependence pattern of the barrier density by a numerical analysis example of the Cauchy problem solution in which the parameter simulating values and the initial defect densities $C_{i}^{(0)}=5 \cdot 10^{13} \mathrm{~cm}^{-3}$ (they are pointed out in the figure captures following then) are used. The numerical solution results of the Cauchy problem (2.6), (2.7) reduced to the dimensionless form attached to the parameter simulating values are shown in Fig.2.1.

Here it is shown the dependence typical behavior of the barrier relative densities of the vacancy $C_{1} / C_{0}$ and interstitial $C_{2} / C_{0}$ types on the relative irradiate dose $\tau / \tau_{0}$ where $C_{0}$ is a measure scale of the defect densities (here it is equal $10^{15} \mathrm{~cm}^{-3}$ ). The measure units are accepted relative to the selected scale $\tau_{0}$ (fluence) the numerical value of which is determined by a specific problem (it is convenient to select a minimal fluence of the specific problem as the dose measure scale; for instance, in the ion irradiation the value of $\tau_{0}$ can be equal $10^{14} \mathrm{ion} / \mathrm{cm}^{2}$ or $10^{22} \mathrm{n} / \mathrm{m}^{2}$ as the contemporary neutron fluence). This dependence 
pattern is universal and independent of the selected measure scales of the specific physics parameters. In increasing dose it is happened the saturation of the material by the radiation - induced barriers (their densities do not increased far more).
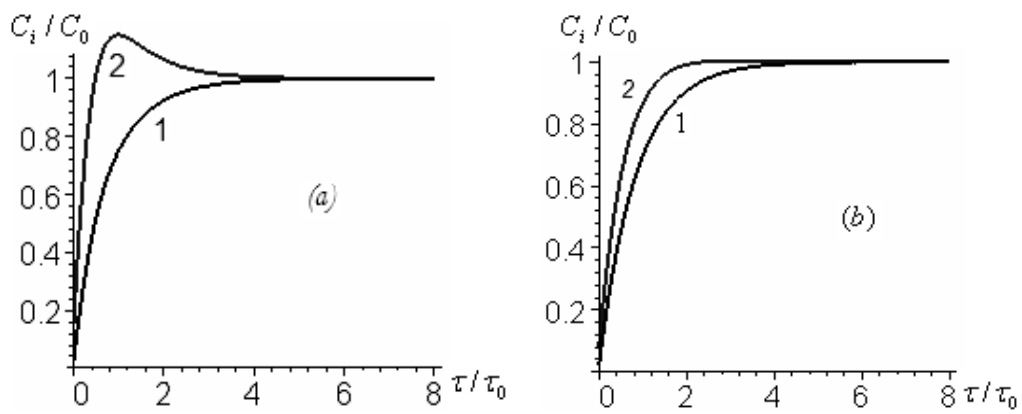

Fig. 2.1. Dependences of relative barrier densities of vacancy type (the curve 1) and interstitial type (the curve 2) on irradiate dose at fixed values of the dimensionless parameters $\left(K_{1} \tau_{0}\right) / C_{0}=2.5, \tau_{0} / \tau_{1}=1.1,\left(K_{2} \tau_{0}\right) / C_{0}=1, \tau_{0} / \tau_{2}=1.5$, and different values of the recombination coefficient: $(a)-\gamma_{12} C_{0} \tau_{0}=0.9,(b)-\gamma_{12} C_{0} \tau_{0}=0.1$ where $C_{0}, \tau_{0}$ are the measure scales of barriers and dose (fluence) respectively the concrete selection of that is conditioned by the concrete problem.

The numerical analysis shows that at $K_{1}>K_{2}$ and small doses, the barrier density of the vacancy type exceeds the one of the interstitial type. At opposite inequality, a situation becomes reverse that is natural as it physically means that increasing the irradiation intensity leads to enlarging a number both the vacancy and the interstitial barriers. Upon that the higher the velocity of forming the radiation barriers of any type is the larger their volume density. It is necessary to mark that the vacancy barrier density usually is higher than the interstitial barrier density in the real materials near a sample surface irradiated.

The numerical analysis also shows that increasing the mutual recombination coefficient $\gamma_{12}$ leads to decreasing the saturation values of the barrier densities. It leads as well to changing the dose dependence pattern of the barrier density of the corresponding type that becomes the monotonic quantity (see Fig.2.1, a) and b)).

Substitution of the found numerical solution of the system (2.7) to Eq (2.3) allows getting the dose dependences of the material yield strength increment. For plotting these dependence figures, it is convenient to represent $\mathrm{Eq}(2.3)$ as follows

$$
\begin{gathered}
\Delta \sigma=\Delta \sigma_{\infty}^{0}\left\{\left(d_{1} C_{1} / d C_{0}\right)^{1 / 2}+\left(d_{2} C_{2} / d C_{0}\right)^{1 / 2}\right\}, \\
\Delta \sigma_{\infty}^{0}=\alpha \mu b\left(C_{0} d\right)^{1 / 2}
\end{gathered}
$$

where $d$ is an average size of the barrier clusters of all types (here it can be determined as half-sum of $d_{1}$ and $d_{2}$ ). 
The results of numerical modeling the behavior of the yield strength are represented in Fig. 2.2. where the typical dose dependences of the yield strength increment are shown on the base of the obtained Eqs (2.8) and (2.9) (in relative units) at the model parameter values. The corresponding numerical analysis shows that the yield strength gets the saturation quickly enough. The typical monotonic form of the dose saturation plots of the yield strength does not change virtually in a broad enough interval of the model parameter values satisfying to the existence condition of the Cauchy problem (2.5), (2.6) solution.

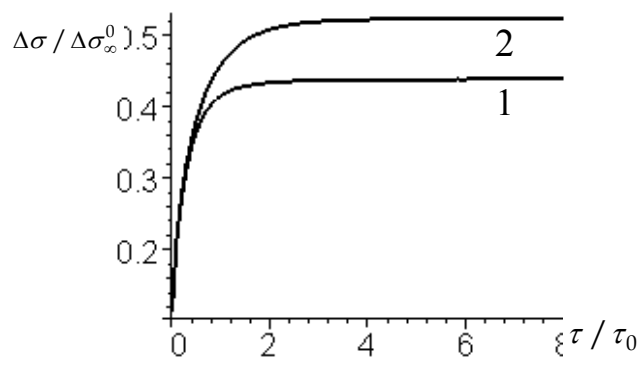

Fig. 2.2. Dependences of the relative yield strength increment on irradiate dose at fixed the nondimensional parameters $\left(K_{1} \tau_{0}\right) / C_{0}=2.5, \tau_{0} / \tau_{1}=1.1,\left(K_{2} \tau_{0}\right) / C_{0}=1, \tau_{0} / \tau_{2}=1.5$, and different values of the recombination coefficient: (1) $\gamma_{12} C_{0} \tau_{0}=0.9$, (2) $\gamma_{12} C_{0} \tau_{0}=0.1$ in relative units.

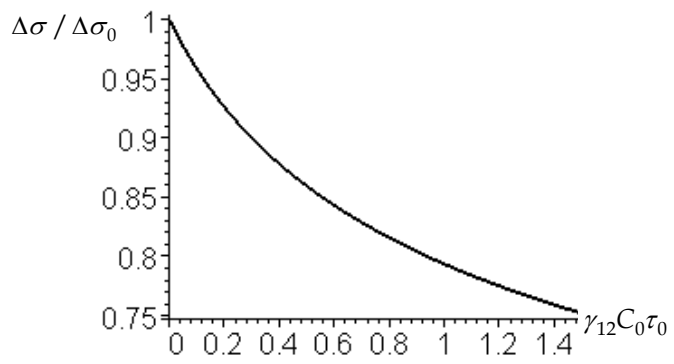

Fig. 2.3. Dependences of the relative yield strength increment on the recombination coefficient at fixed irradiate dose $\tau / \tau_{0}=4$ and the rest parameter values as in Fig. 2.2.

Besides, on the base of the numerical analysis, it is obtained that a growth of the mutual recombination coefficient $\gamma_{12}$ leads to decreasing the saturation value of the yield strength. It is shown in Fig.2.3. where the dependence of the relative yield strength increment $\Delta \sigma / \Delta \sigma_{0}$ on the mutual recombination coefficient at fixed dose is shown (here $\Delta \sigma_{0}$ is the yield strength increment value at $\gamma_{12}=0$ that is under the condition of total neglecting interaction between the vacancy and interstitial barriers). It is well seen that the relative yield strength increment decreases with the growth of the mutual recombination coefficient, this dependence being nonlinear and monotonic.

A stationary point of the systems (2.7) determines the saturation values of the barrier density: 


$$
\begin{gathered}
C_{1}^{(s)}=\frac{K_{1}}{K_{1} V_{1}+\gamma_{12} C_{2}^{(s)}}, \\
C_{2}^{(s)}=C_{a}\left(1+\sqrt{1+\frac{C_{b}}{C_{a}}}\right),
\end{gathered}
$$

where

$$
C_{a}=\frac{\left(K_{2}-K_{1}\right) \gamma_{12}-K_{1} K_{2} V_{1} V_{2}}{2 \gamma_{12} K_{2} V_{2}}, C_{b}=\sqrt{\frac{K_{1} V_{1}}{\gamma_{12} V_{2}}} .
$$

The saturation values of the barrier density (2.10) and (2.11) are achieved quickly enough (see Fig.2.1.).

In the case when the recombination of the vacancy and interstitial barriers is negligibly small the equations of the system (2.7) become independent at $\gamma_{12}=0$. If to suppose in addition that the radiation barriers are virtually absent at the initial time $C_{i}^{(0)}=0,(i=1,2)$ then as a result the known expression of the volume barrier density is obtained [3]:

$$
C_{i}(\tau)=\frac{1}{V_{i}}\left(1-e^{-\tau / \tau_{i}}\right), \quad i=1,2,
$$

where $\tau_{i}=\left(K_{i} V_{i}\right)^{-1}$. Substitution of Eq (2.12) to Eq (3) gives the expression of the yield strength increment at $\gamma_{12}=0$ :

$$
\Delta \sigma_{0}=\Delta \sigma_{1}^{(s)}\left(1-e^{-\tau / \tau_{1}}\right)^{1 / 2}+\Delta \sigma_{2}^{(s)}\left(1-e^{-\tau / \tau_{2}}\right)^{1 / 2},
$$

where $\Delta \sigma_{i}^{(s)}=\alpha_{i} u b\left(d_{i} / V_{i}\right)^{1 / 2}, i=1,2$. In this case, at large doses it is followed from Eq (2.13) that. $\Delta \sigma_{0}=\Delta \sigma_{1}^{(s)}+\Delta \sigma_{2}^{(s)}$

It should be noted that at small doses it is followed the well known law of Eq (2.2) at $n=1 / 2$ :

$$
\Delta \sigma_{0}=a(\Phi t)^{1 / 2}
$$

where $a=\mu b\left\{\alpha_{1}\left(K_{1} d_{1}\right)^{1 / 2}+\alpha_{2}\left(K_{2} d_{2}\right)^{1 / 2}\right\}$.

In the absence of the mutual recombination of the vacancy and interstitial barriers but at the arbitrary initial barrier densities and the initial conditions (2.6) and at $\gamma_{12}=0$, the solution of the equation system (2.7) leads to the expression

$$
C_{i}(\tau)=\frac{1}{V_{i}}\left\{1+\left(C_{i}^{(0)} V_{i}-1\right) e^{-K_{i} V_{i} \tau}\right\}, i=1,2
$$

Substituting Eq (2.15) into (2.3) it can be obtained the expression for the yield strength increment at $\gamma_{12}=0$ and the arbitrary initial barrier densities:

$$
\Delta \sigma_{0}=\Delta \sigma_{1}^{(s)}\left\{1+\left(C_{1}^{(0)} V_{1}-1\right) e^{-K_{1} V_{1} \tau}\right\}^{1 / 2}+\Delta \sigma_{2}^{(s)}\left\{1+\left(C_{2}^{(0)} V_{2}-1\right) e^{-K_{2} V_{2} \tau}\right\}^{1 / 2}
$$


$\mathrm{Eq}$ (2.16) is used to plot the yield strength dependence on the intensity quantity of the vacancy and interstitial barrier recombination at fixed dose in Fig. 2.3.

\subsection{Dose saturation features of the yield strength subject to the effects of clustering barriers}

Let us consider now the main contribution to the radiation hardening is given by the effects of forming the clusters of two barriers of the same type and the mutual recombination of the vacancy and interstitial barriers is negligibly small. Therefore, $\gamma_{12}<<\gamma_{i}, i=1,2$, and in every equation of the system (2.5) it can be neglected by the last terms of $\gamma_{12} C_{1} C_{2}$. After this, the system equations become independent and therefore in what follows the indexes 1 and 2 of notations can be omitted.

Further we consider the contribution to the radiation hardening only from the barriers of the same type (either vacancy or interstitial) and then instead Eqs (2.3) and (2.4) the next expression is used

$$
\Delta \sigma=\alpha \mu b(C d)^{1 / 2},
$$

where $\alpha$ is the parameter characterizing the barrier intensity (a fixed quantity for some of barrier types), $C$ the volume barrier density of the same type, $d$ their average size.

In this case it is convenient to go to single equation of the system (2.5):

$$
\frac{d C}{d \tau}=K\left(1-V C-\gamma C^{2}\right)
$$

where $\gamma_{i}$ of Eqs (2.5) is changed by the new notation according to the relationship $\gamma_{i}=K \gamma$ and all of the indexes are omitted.

The solution of $\mathrm{Eq}(2.18)$ with the initial condition $\mathrm{C}(0)=0$ at $\gamma>0$ takes the form

$$
C(\tau)=\frac{1}{2 \gamma}\left\{q \operatorname{th}\left(\frac{\tau}{\tau^{\prime}}+\varphi\right)-V\right\}
$$

where $\tau^{\prime}=2 / K q, \quad q=\sqrt{V^{2}+4 \gamma}, \quad \varphi=\operatorname{Arth} \frac{V}{q}=\frac{1}{2} \ln \frac{q+V}{q-V}$. The found expression

describes the dependence of the volume radiation-induced barrier density on irradiate dose (fluence) $\tau=\Phi t$. The typical plot of the dependence (2.19) is shown in Fig.2.4. at the different values of barrier clusterization intensity and the fixed rest parameters in the relative units (as stated above).

Substituting Eqs (2.19) into Eqs (2.17) we obtain the dose dependence of the yield strength increment:

$$
\Delta \sigma=\Delta \sigma_{\infty}^{0} \frac{1}{\sqrt{2 \gamma C_{0}}}\left\{q \operatorname{th}\left(\frac{\tau}{\tau^{\prime}}+\varphi\right)-V\right\}^{1 / 2}
$$




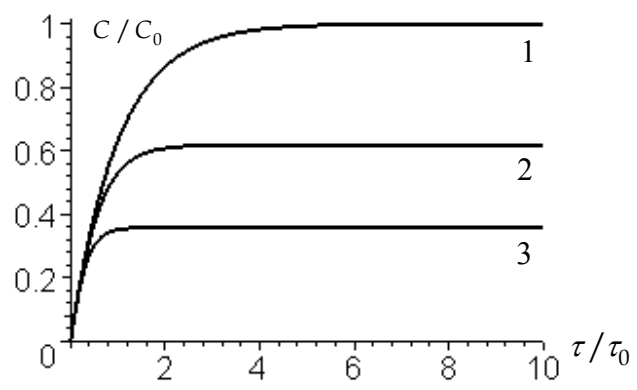

Fig. 2.4. Dose dependences of the volume barrier density (2.19) at the fixed parameter values $\left(K \tau_{0}\right) / C_{0}=1, C_{0} V=1$ and the different values of clusterization intensity: (1) $C_{0}^{2} \gamma=0$, (2) $C_{0}^{2} \gamma=1$, (3) $C_{0}^{2} \gamma=5$ in relative units.

In the present model, it is supposed that the average barrier size is a weakly changing function of irradiate dose. The typical plot of the dependence (2.20) is shown in Fig. 2.5 at the different values of barrier clusterization intensity and the fixed rest parameters.

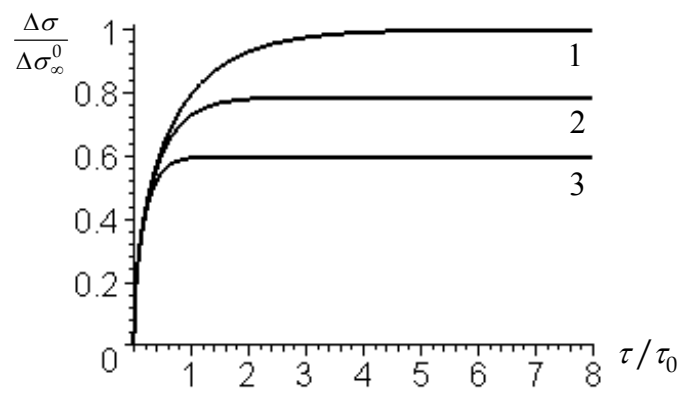

Fig. 2.5. Dose dependences of the relative yield strength increment (2.20) at the fixed parameter values $\left(K \tau_{0}\right) / C_{0}=1, C_{0} V=1$, and the different values of clusterization intensity: (1) $C_{0}^{2} \gamma=0$, (2) $C_{0}^{2} \gamma=1$, (3) $C_{0}^{2} \gamma=5$ in relative units.

It follows from (2.19) that at low irradiate doses that is when $\tau<<\tau^{\prime}$ (accordingly (2.19) it corresponds to low density of radiation defects) the dependence of the volume density of the radiation-induced barriers on irradiate dose is linear: $C=(q+V) q K \Phi t$. Substituting this expression into (2.17) we obtain the dependence of $\Delta \sigma=a(\Phi t)^{1 / 2}$ where $a=\alpha \mu b\{(q+V) q K d\}^{1 / 2}$.

At high irradiate doses that is when $\tau>>\tau^{\prime}$ the volume density of the radiation-induced barriers is saturated and tends to the constant $C_{\infty}=(q-V) / 2 \gamma$. This value of the volume density is the stationary point of Eq (2.18). Substituting this expression into Eqs (2.17) we obtain the saturation value of the yield strength:

$$
\Delta \sigma_{\infty}=\Delta \sigma_{\infty}^{0}\left(\frac{q-V}{2 \gamma C_{0}}\right)^{1 / 2}
$$


where $\Delta \sigma_{\infty}^{0}$ is determined by Eq (2.9).

If the clusterization effects can be neglected (as well as the mutual annihilation of the barrier of the different types has been already neglected too) then it can be obtained from Eq (2.19) the known expression of the volume barrier density as Eq (2.12). This dependence corresponds to the curve 1 in Fig.2.4.

After substituting such volume density into Eq (2.17) it is obtained the dose dependence of the yield strength in the case when the barrier clusterization makes negligibly small contribution to the velocity of forming the radiation - induced barriers [3]:

$$
\Delta \sigma^{0}=\Delta \sigma_{\infty}^{0}\left(\frac{1-e^{-\tau / \bar{\tau}}}{C_{0} V}\right)^{1 / 2},
$$

where $\bar{\tau}=(K V)^{-1}$. This dependence corresponds to the curve 1 in Fig.2.5. Hence it follows at small irradiate doses the well known law as $\Delta \sigma=a(\Phi t)^{1 / 2}$ where now $a=\alpha \mu b(K d)^{1 / 2}$.

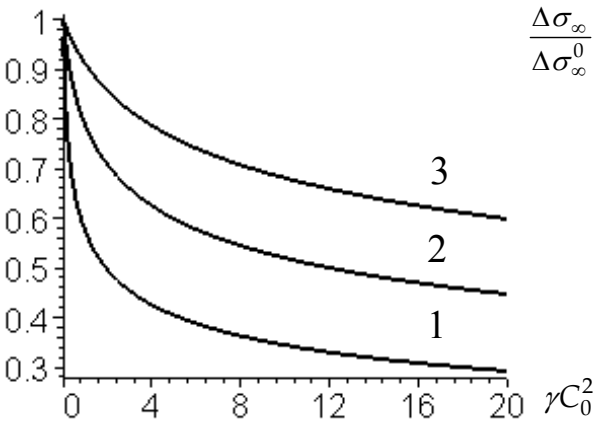

(a)

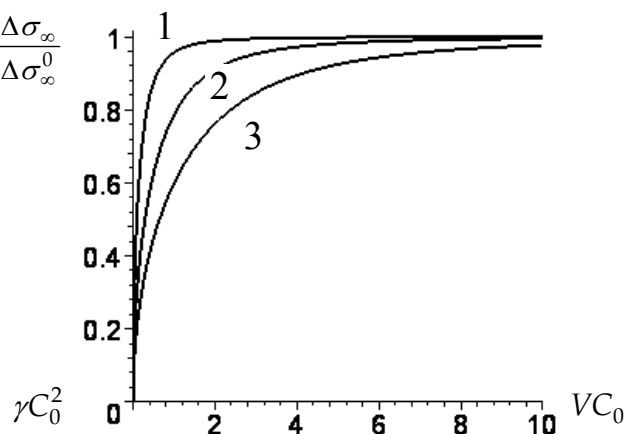

(b)

Fig. 2.6. Dependences of the relative yield strength increment saturation (2.21) at the fixed parameter values $\left(K \tau_{0}\right) / C_{0}=1$ :

(a) dependence on nondimensional intensity of the barrier clusterization $C_{0}^{2} \gamma$ at different values of their volumes: (1) $C_{0} V=0.4$, (2) $C_{0} V=1$, (3) $C_{0} V=2$ in relative units.

(b) dependence on $V$ at different values of the clusterization intensity: (1) $C_{0}^{2} \gamma=0.1$, (2) $C_{0}^{2} \gamma=1$, (3) $C_{0}^{2} \gamma=5$ in relative units.

On the base of the obtained dependence of the yield strength saturation by Eq (2.21) on the barrier recombination intensity, it can be drawn the next conclusion. The saturation quantity of the material yield strength at fixed dose decreases monotonically with increasing the intensity of radiation - induced barrier clusterization. This behavior is shown in Fig.2.6. where the plots of Eq (2.21) are presented.

\subsection{Discussion of experiment data}

As known, since 1988 the EU, USA, Japan and Russia joint works have been fulfilled within the intergovernmental agreement approved by IAEA (International Atomic Energy 
Agency). According to technological design of the thermonuclear reactor ITER, one of main constructional material is austenitic steel 316(N)-IG [6].

In the Ref [9], it is developed the model of the radiation hardening of the concrete irradiation material, 316(N)-IG steel. This model is based on the equation to be equivalent to Eq (2.18) for the potential barrier density $C$ the role of which plays the stacking fault tetrahedral observed by electron microscopy as black dots. Experiment reveals that the concentration of these barriers grows with increasing irradiation dose.

Authors of the work [9] make a comparison with experimental data for 316(N)-IG steel on the base of the equation analogous to above Eq (2.22) to be the particular case of Eq (2.18) that is not taking into account of the barrier annihilation. They receive relatively good fit with the experimental data in temperature ranges $20-150$ and $230-300^{\circ} \mathrm{C}$. For the higher temperatures $\left(330-400^{\circ} \mathrm{C}\right)$ this equation does not obey an adequate description of the yield strength and ultimate stress increment. To describe the experimental data the authors of Ref [7] fit an exponent in the dependence of the form (2.17) pointing out this exponent to be varied approximately in the interval $1.4-2.7$ for the best agreement with the experimental data. It is possible that accounting the barrier recombination of the different types leads to invariability of the exponent $1 / 2$ in $\mathrm{Eq}$ (2.17).

In the work [8], the equation analogous to Eq (2.22) it is used for fitting the experimental data for strength and ductility of corrosion - resisting austenitic 06X18H10T steel irradiated by the WWER - 440 reactor up to damaging dose $\sim 21$ d.p.a. at different testing temperatures. Authors of Ref [8] find out that the radiation hardening saturation of 06X18H10T steel irradiated in WWER-440 reactors takes place at the damage dose of $\sim 10 \div$ 15 d.p.a. $\left(1 \div 1,5 \cdot 10^{26} \mathrm{n} / \mathrm{cm}^{2}\right)$.

\section{Effect of secondary processes on material hardening under low temperature radiation}

\subsection{Formulation of the secondary process contribution model}

The base of the model is a barrier mechanism of the radiation hardening [2] according to that the yield strength increment can be represented by the sum of barrier contributions of different types (see Eq (2.1) of the preceding section).

We consider that the barriers of vacancy and interstitial types make a main contribution to the yield strength increment of a certain material. These barriers play the main role in the hardening at the low temperature irradiation. Therefore, in the proposed model $N=3$; the index values of $i=1$ correspond to the vacancy barriers, $i=2$ do to interstitial ones and $i=3$ do to more large vacancy complexes. Then in this model Eq (2.1) takes the form:

$$
\Delta \sigma=\Delta \sigma_{1}+\Delta \sigma_{2}+\Delta \sigma_{3}
$$

For all of the obstacle types, the metal yield strength increment conditioned by dislocation deceleration is described by the Eq (2.4) (see the preceding section).

Under irradiation, development of radiation defect clusters (barriers) of different types occurs in a region of a primary knocked-on atom. It is proposed that the interstitial barriers have considerably smaller sizes and leave the damage region of a sample sooner than the 
vacancy barriers do. In connection with this, we formulate the phenomenological model that is based on the equation system for volume densities of the radiation-induced nonequilibrium vacancy $C_{1}$ and interstitial barriers $C_{2}$ and more large complexes $C_{3}$ developed by bimolecular mechanism of the vacancy barriers:

$$
\left\{\begin{array}{l}
\frac{\partial C_{1}}{\partial \tau}=D_{1} \Delta C_{1}+K_{1}-C_{1} / \tau_{1}-\gamma_{12} C_{1} C_{2}-\gamma_{1} C_{1}^{2} \\
\frac{\partial C_{2}}{\partial \tau}=D_{2} \Delta C_{2}+K_{2}-C_{2} / \tau_{2}-\gamma_{12} C_{1} C_{2}-\gamma_{2} C_{2}^{2} \\
\frac{\partial C_{3}}{\partial \tau}=D_{3} \Delta C_{3}+\gamma_{1} C_{1}^{2}-C_{3} / \tau_{3} .
\end{array}\right.
$$

Here $\tau=\Phi t, \Phi$ is the particle flux, $t$ irradiation time, $K_{i}, i=1,2$, the intensities of forming the radiation - induced vacancy and interstitial barriers, $\gamma_{i}$ are the coefficients of barrier recombination and characterize forming the clusters of acceptable barrier type (it can be named as clusterization coefficients), the coefficients $\tau_{i}^{-1}$ can be represented by the form: $\tau_{i}^{-1}=K_{i} V_{i}$, where $V_{i}$ are the effective volumes of interaction of the certain barriers with each other, $\gamma_{12}$ the coefficient of mutual recombination of the annihilating vacancy and interstitial barriers. It can be valued as follows:

$$
\gamma_{12}=\frac{4 \pi r\left(D_{1}+D_{2}\right)}{\Omega} e^{-\frac{E_{r}}{k_{B} T}},
$$

where $\Omega$ atom volume, $E_{r}$ activation energy of recombination of vacancy and interstitial barriers, $r$ recombination radius, $T$ test temperature, $k_{B}$ Boltzmann constant, $D_{i}$ diffusion coefficients of the non-equilibrium barriers of the given types: $D_{i}=D_{i 0} \exp \left(-E_{i} / k_{B} T\right)$, $i=1,2$, where $E_{i}$ energy of activation and migration of respective barriers, $D_{i 0}=a^{2} v, a$ and $v$ are length and barrier jumping frequency for migration, respectively.

As material structure changes go under irradiation for times large in comparison with relaxation time of point defects then only diffusion barrier processes are considered to be very slow and therefore we neglect diffusion terms in the equations of the system (3.2). In addition, we study evolution of barrier volume densities in time considering their distributions are spatially homogeneous. In this case, the system (3.2) takes the form:

$$
\left\{\begin{array}{l}
\frac{\partial C_{1}}{\partial \tau}=K_{1}-C_{1} / \tau_{1}-\gamma_{12} C_{1} C_{2}-\gamma_{1} C_{1}^{2} \\
\frac{\partial C_{2}}{\partial \tau}=K_{2}-C_{2} / \tau_{2}-\gamma_{12} C_{1} C_{2}-\gamma_{2} C_{2}^{2} \\
\frac{\partial C_{3}}{\partial \tau}=\gamma_{1} C_{1}^{2}-C_{3} / \tau_{3} .
\end{array}\right.
$$

In this equation system, the first two equations coincide with the system (2.5) completely (see the preceding section). 
The third equation of the system (3.3) describes redistribution of divacancy barrier complexes. Contribution to the hardening due to divacancy barriers is determined by only vacancy barrier density. The kinetic coefficients $\tau_{3}^{-1}$ and $\gamma_{1}$ characterize intensities of breakdown of vacancy clusters and development of divacancy clusters. Effect of these contributions are appreciable if intensity of secondary processes of developing divacancy complexes predominates over their breakdown.

To find the volume densities of the nonequilibrium barriers it is necessary to set up their initial values:

$$
C_{i}(0)=C_{i}^{(0)}, i=1,2,3
$$

Thus, the mathematical formulation of the model proposed in this section is the Cauchy problem for the system of nonlinear differential equations (3.3) with initial conditions (3.4). The volume barrier densities found as a result of solution of the Cauchy problem (3.3), (3.4) are to be inserted into Eq (2.4) that determines the total yield strength increment (3.1).

\subsection{Numerical analysis of the model results}

The model values of parameters (are given in captures of Figures) and the initial conditions $C_{i}^{(0)}=5 \cdot 10^{13} \mathrm{~cm}^{-3}$.are used to fulfill numerical analysis of the Cauchy problem (3.3), (3.4). The results of the numerical solution of the Cauchy problem (3.3), (3.4) (reduced to the dimensionless form) are represented on Fig.3.1. at the indicated parameter values.

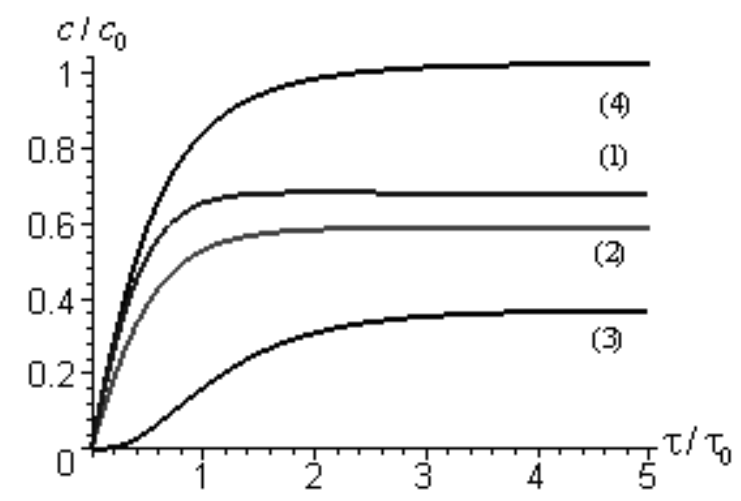

Fig. 3.1. Dependences of relative barrier density of vacancy type (1), interstitial type (2), vacancy complexes (3) and their total density (4) on dose at fixed values of dimensionless parameters $\left(K_{1} \tau_{0}\right) / C_{0}=1.5,\left(K_{2} \tau_{0}\right) / C_{0}=1, \tau_{0} / \tau_{1}=1, \tau_{0} / \tau_{2}=0.5, \tau_{0} / \tau_{3}=1.25$, $\gamma_{12} C_{0} \tau_{0}=0.9, C_{0}^{2} \gamma_{1}=C_{0}^{2} \gamma_{2}=1$.

Here is shown the specified form of dose dependences of relative densities for vacancv barriers $C_{1} / C_{0}$, and interstitial barriers $C_{2} / C_{0}$, and more large vacancy complexes $C_{3} / C_{0}$ where $C_{0}$ is a measure scale of barrier density taken to be equal $10^{15} \mathrm{~cm}^{-3}$ in this case on dose $\tau / \tau_{0}$ in units measured by the scale $\tau_{0}$ (it is convenient to select a minimal fluence of 
the specific problem as the dose measure scale; for instance, in the ion irradiation the value of $\tau_{0}$ can be equal $10^{14}$ ion $/ \mathrm{cm}^{2}$ or $10^{22} \mathrm{n} / \mathrm{m}^{2}$ in the neutron irradiation and so on, for the specific problem, respectively). These dependence patterns are universal and independent of the selected measure scales of the specific physics parameters. It is shown that the saturation of the material by the radiation - induced barriers takes place with increasing dose.

The numerical solution of the Cauchy problem (3.3), (3.4) permits to obtain the dose dependences of the total increment of material yield strength which is convenient to represent for construction of graph as follows

$$
\Delta \sigma=\Delta \sigma_{\infty}^{0} \sum_{i=1}^{3} \sqrt{d_{i} C_{i} / d C_{0}},
$$

where $\Delta \sigma_{\infty}^{0}$ is determined by $\mathrm{Eq}$ (2.9) (see the preceding section), $d$ average size of barrier cluster over all of types. The results of numerical modeling the behavior of the yield strength increment are represented in Fig. 3.2.

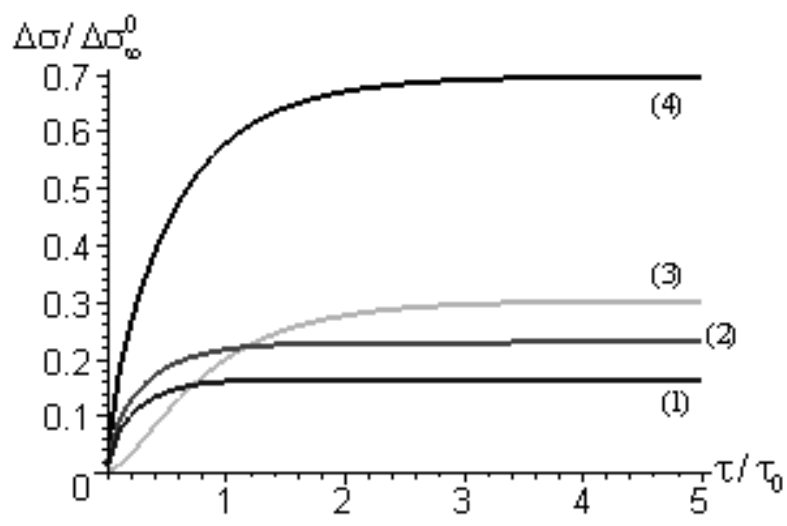

Fig. 3.2. Dose dependences of barrier contributions of vacancy type (1), interstitial type (2), vacancy complexes (3) and their total density (4) to yield strength increment at fixed values of parameters the same as in Fig.3.1. and $d_{1} / d=0.016, d_{2} / d=0.035, d_{3} / d=0.097$.

The numerical analysis shows that the yield strength increment gets the saturation quickly enough. The typical monotonic form of the dose saturation plots of the yield strength increment does not change virtually in a broad enough interval of the model parameter values satisfying to the existence condition of the Cauchy problem (3.3), (3.4) solution.

It is should be noted though the vacancy complexes have lower concentration in comparison with vacancies and interstitial atoms they, due to their larger sizes, contribute more considerably to yield strength increment at dose build-up. 


\subsection{Secondary reaction contribution analysis}

Let us consider the case when secondary reactions play a main role that is barrier recombination goes less intensively than developing barrier clusters. In this extreme case, we consider $\gamma_{12}<<\gamma_{1}$ and $\gamma_{12}<<\gamma_{2}$. Then the second equation of the system (3.3) becomes independent and coinciding formally with the first equation. In the result, the system (3.3) consists of two equations:

$$
\left\{\begin{array}{l}
\frac{\partial C_{1}}{\partial \tau}=K_{1}-C_{1} / \tau_{1}-\gamma_{1} C_{1}^{2}, \\
\frac{\partial C_{3}}{\partial \tau}=\gamma_{1} C_{1}^{2}-C_{3} / \tau_{3} .
\end{array}\right.
$$

The first equation of the system (3.6) doesn't contain $C_{3}$. Therefore, it is independent. Its solution with zero initial condition takes the form:

$$
C_{1}(\tau)=C_{a} \tanh \left(\frac{\tau}{\tau_{c}}+\varphi\right)-C_{b},
$$

where $\tau_{c}=2 \tau_{1} / \kappa, C_{a}=\kappa / 2 \tau_{1} \gamma_{1}, C_{b}=C_{a} / \kappa, \kappa=\sqrt{1+4 K_{1} \gamma_{1} \tau_{1}^{2}}, \varphi=\operatorname{Artanh}(1 / \kappa)$. The obtained expression (3.7) describes the dependence of vacancy barrier volume density on dose $\tau=\Phi t$.

When the processes of vacancy barrier clusterization are absent overall $\left(\gamma_{1}=0\right)$ it results from (3.6) $C_{1}(\tau)=K_{1} \tau_{1}\left(1-e^{-\tau / \tau_{1}}\right)$ whence the well known contribution to yield strength increment follows in the case of hardening by the barrier of a single type:

$$
\Delta \sigma_{1}=\Delta \sigma_{\infty}^{0}\left\{d_{1} K_{1} \tau_{1}\left(1-e^{-\tau / \tau_{1}}\right) / d C_{0}\right\}^{1 / 2} .
$$

Substituting Eq (3.7) into the second equation of the system (3.6) its solution can be written as

$$
C_{3}(\tau)=\gamma_{1} \int_{0}^{\tau} e^{(s-\tau) / \tau_{3}} C_{1}^{2}(\tau) d s
$$

Further, this expression is used to analysis the vacancy complex contribution to yield strength increment:

$$
\Delta \sigma_{3}=\Delta \sigma_{\infty}^{0}\left(d_{3} C_{3} / d C_{0}\right)^{1 / 2}
$$

The increasing of saturation quantity of yield strength increment goes with increasing intensity of the clusterization processes that is with increasing parameter $\gamma_{1}$ (Fig.3.3a).

This increasing is nonlinear, enlargement of yield strength increment saturation going less and less considerably with increasing intensity of the clusterization processes.

Growth of saturation quantity of yield strength increment goes with increasing specific times $\tau_{1}$ and $\tau_{3}$ as well (Fig.3.3. $b$ and $c$ ). 


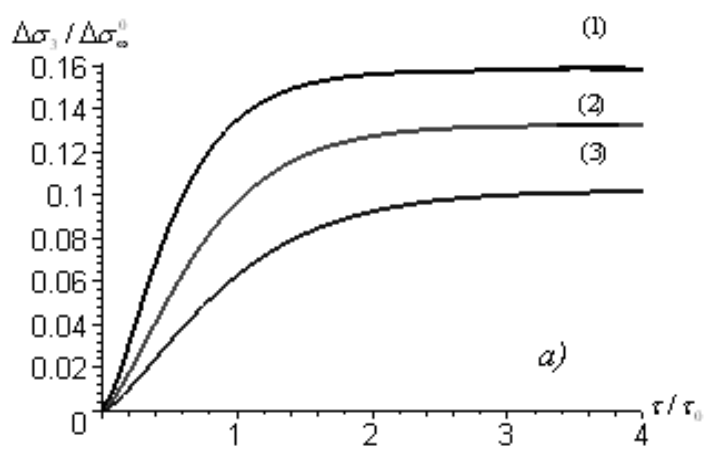

Fig. 3.3. a. Dose dependences of vacancy complex contribution to yield strength increment at fixed values of parameters $\left(K_{1} \tau_{0}\right) / C_{0}=1, d_{3} / d=0.097, \tau_{1} / \tau_{0}=1, \tau_{3} / \tau_{0}=0.4$, (1) $C_{0}^{2} \gamma_{1}=5 ;(2)-C_{0}^{2} \gamma_{1}=1.5 ;(3)-C_{0}^{2} \gamma_{1}=0.5$.
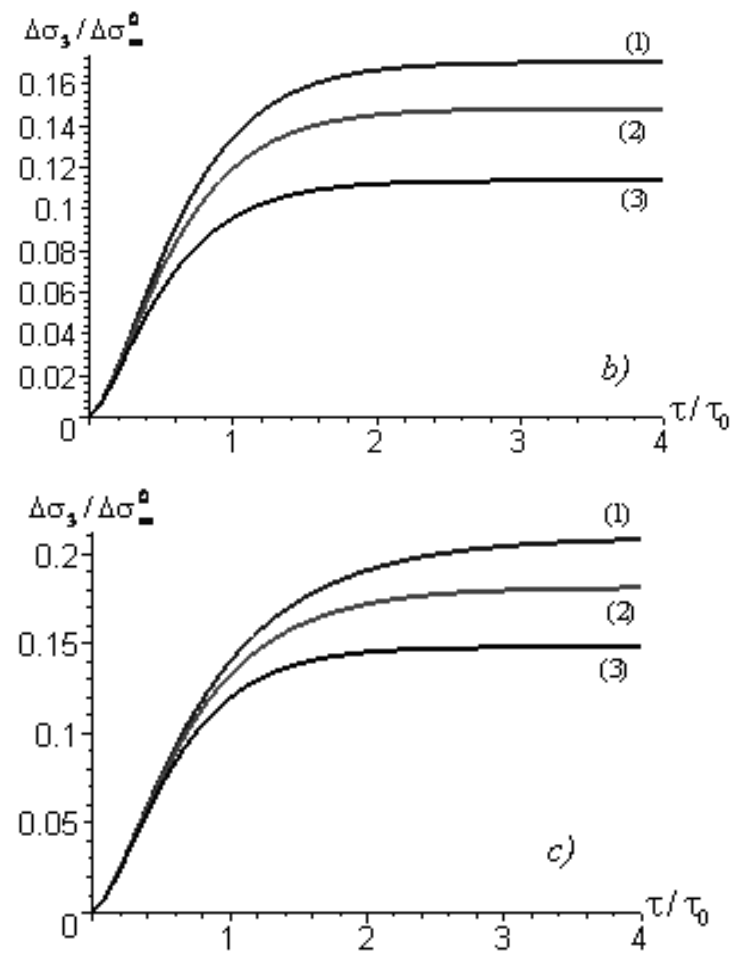

Fig. 3.3. $b$ and c. Dose dependences of vacancy complex contribution to yield strength increment at fixed values of parameters $\left(K_{1} \tau_{0}\right) / C_{0}=1, d_{3} / d=0.097$,

b) $\tau_{3} / \tau_{0}=0.4, C_{0}^{2} \gamma_{1}=3,(1)-\tau_{1} / \tau_{0}=2 ;(2)-\tau_{1} / \tau_{0}=1 ;(3)-\tau_{1} / \tau_{0}=0.5$;

c) $\tau_{1} / \tau_{0}=1, C_{0}^{2} \gamma_{1}=3,(1)-\tau_{3} / \tau_{0}=0.8 ;(2)-\tau_{3} / \tau_{0}=0.6$; (3) $-\tau_{3} / \tau_{0}=0.4$. 


\section{Phenomenological model of yield strength dependence on the temperature of irradiated materials}

\subsection{Temperature intervals of radiation embrittlement with taking into account two components of material flow stress}

The results of experimental studying radiation embrittlement effects and the temperature dependences of such durable material characteristics as specific elongation and yield strength have been given in a series of the works [10-15]. In Refs [12, 15], it is shown that a deformation process connected with dislocation collective behavior in irradiated deformed materials is characterized by availability of the different structure deformation levels.

As known under irradiation material plastic properties undergo strong changes. In particular a radiation embrittlement phenomenon takes place [11]. Upon that plastic properties of irradiated materials depend essentially on temperature. It is interesting to analyze phenomena of radiation embrittlement and radiation hardening of reactor materials with taking into account their durable characteristics on temperature.

To analyze radiation embrittlement it is necessary to take into account availability of two components of material flow stress $(\sigma)$ : the thermal (thermo activated) component $\sigma^{*}$ created by short - rang forces and the athermal one $\sigma_{\mu}$ determined by long - range forces of slowing - down dislocations and no experiencing influence of temperature. These components are shown on the plot of a temperature dependence of material flow stress (Fig.4.1.).

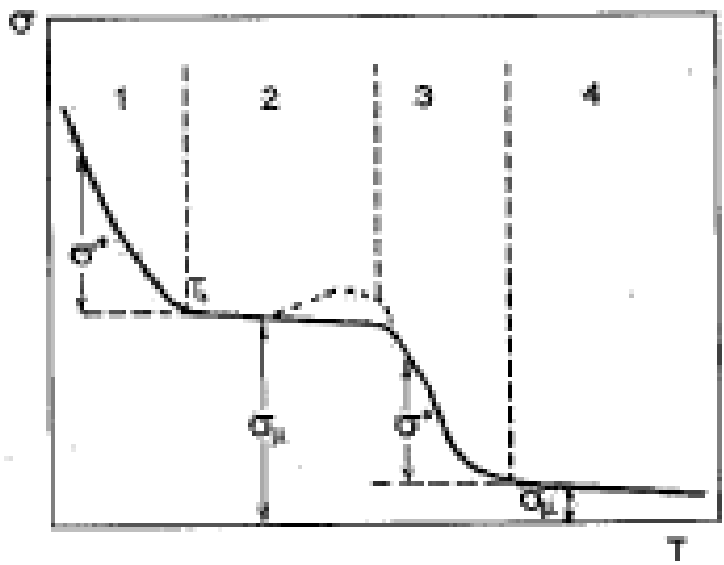

Fig. 4.1. Generalized scheme of temperature dependence of flow stress in polycrystalline materials. Area 1 corresponds to low temperature range $\mathrm{T}<0,15 \mathrm{~T}_{\mathrm{m}}$; area 2 is characterized by athermal component $\sigma_{\mu}$ up to $\sim 0,45 \mathrm{~T}$ m; area 3 corresponds to thermo activated component of flow stress; area 4 corresponds to second athermal plateau $\sigma_{\mu} \sim \mathrm{G}$.

The first activated area (1) on Fig. 4.1. covers a low temperature interval $T \leq 0,15 \cdot T_{\mathrm{m}}$ in which a activative volume quantity of plastic deformation is as $b^{3}$ where $b$ is the Burgers vector modulus. This corresponds to a microscale level of dislocation interactions that is realized 
by point kinetics processes of dislocations. The area (2) on Fig. 4.1. is characterized by availability of athermal component $\sigma_{\mu}$ which is mainly determined by long - range elastic internal stresses forming due to interaction of dislocations moving in parallel or crossing sliding planes. At temperatures of $T \geq 0,45 \cdot T_{\mathrm{m}}$ (the area (3) of Fig. 4.1.), edge dislocation creeping conditioned by diffusion processes and forming crew dislocation jogs are determined by the thermo activated flow stress component. In the area (4) of Fig. 4.1., intensification of grain boundary processes of plastic deformation takes place and forms a second athermal plateau $\sigma_{\mu} \sim G$.

Connection of $\sigma^{*}$ and $\sigma_{\mu}$ changes with temperature dependence of radiation embrittlement in a wide enough experience temperature interval including certain areas shown on Fig. 4.1. can be studied by a method of modeling neutron irradiation action by relativistic electron beams with energies exceeding nuclear reaction threshold (so called $((e, \gamma)$ - beams). Such irradiation as well as reactor one leads to forming different radiation defects ( for instance, defects of diclocation loop type) besides nuclear reaction products) [3, 16].

Main preference of such beams is a possibility to create for short time (for some of hours) radiation damages equivalent to ones obtained for some of years of irradiation in reactors. Besides modeling experiments can be fulfilled under severely controlled conditions that has paramount importance to clear up mechanisms of phenomena in nuclear and thermonuclear reactor materials under exploitation.

The beams of electrons and $\gamma$ - quanta having a large track length in materials make it possible to create homogeneous radiation damages in samples assigned for investigating mechanical properties. Investigations of mechanical prosperities of materials irradiated by $(e, \gamma)$ - beams showed availability of their employment for modeling reactor damages and selection of construction material [17].

When high energy electrons get through substance an electromagnetic avalanche develops. In increasing electron penetration depth in to a material sample a number of avalanche particles increases, energy of electron decreases and the X-ray bremsstrahlung increases.

Irradiation of materials by high energy electrons leads to accumulation of large amount of helium due to secondary $(\gamma, \alpha)$ - reactions, which is accountable for high temperature radiation embrittlement. The unique feature of $(e, \gamma)$ - beams is a possible to receive samples with distinct ratio of helium accumulation rate to rate of forming displacements at the same experiment.

Changing elastic and inelastic properties of polycrystalline materials are caused by lattice damages under irradiation and their next interaction with dislocations. Diffusion of point defects plays important role in process of pinning dislocations in connection with that it can be obtained significant information about radiation defects investigating influence of experience temperature on a quantity of radiation damage.

\subsection{Formulation of the model}

We consider the model in which the temperature dependence can be interpreted as a result the of phase transition between two plastic deformation structure levels that is characterized by specific values of the athermal stress component. 
Changing yield strength $\sigma$ in dependence on temperature $T$ is characterized by derivative $d \sigma / d T$. As the phase transition is considered between two plastic deformation structure levels then this function must take the form that can be approximated by a parabolic dependence on $\sigma$. Such dependence has to be equal zero when yield strength coincides with theoretical quantities of the first (high temperature) $\sigma_{1 \mu}^{\text {th }}$ and the second (low temperature) $\sigma_{2 \mu}^{t h}$ athermal plateau. As a result, it can be written a phenomenological equation of the structure phase transition in question as follows

$$
\frac{d \sigma}{d T}=\frac{\left(\sigma_{1 \mu}^{t h}-\sigma\right)\left(\sigma_{2 \mu}^{t h}-\sigma\right)}{\left(\sigma_{1 \mu}^{t h}-\sigma_{2 \mu}^{t h}\right) \Delta T_{0}},
$$

where $\Delta T_{0}$ - the specific temperature interval in that yield strength increases occur on the magnitude of the thermo activated component of flow stress.

Solution of Eq. (4.1) takes the form:

$$
\sigma(T)=\frac{\sigma_{1 \mu}^{t h}+\sigma_{2 \mu}^{t h}}{2}-\frac{\sigma_{1 \mu}^{t h}-\sigma_{2 \mu}^{t h}}{2} \tanh \left(\frac{T-T_{c}}{2 \Delta T_{0}}\right),
$$

where $T_{c}$ is temperature corresponding to the average value of athermal stresses of the high temperature and low temperature plateau.

To describe the yield strength experiment dependences of the irradiated materials on temperature it is convenient to rewrite Eq. (4.2) as follows

$$
\sigma(T)=\sigma_{c}-\sigma_{m} \tanh \left(\frac{T-T_{c}}{2 \Delta T_{0}}\right),
$$

where $\sigma_{c}=\left(\sigma_{1 \mu}^{\text {th }}+\sigma_{2 \mu}^{\text {th }}\right) / 2, \sigma_{m}=\left(\sigma_{1 \mu}^{\text {th }}-\sigma_{2 \mu}^{\text {th }}\right) / 2$.

Empirical parameters $\sigma_{c}, \sigma_{m}, \Delta T_{0}, T_{c}$ of the model have next phenomenological meaning. Temperature $\Delta T_{0}$ is connected with activation energy $Q_{e}$ of the plastic deformation transition on a higher structure level after that the material goes to a stage of radiation embrittlement: $Q_{e}=\Delta T_{0} k_{B} / 2$ where $k_{B}$ is Boltzmann constant. Parameters $\sigma_{c}$ and $T_{c}$ are stress and temperature of the transition, respectively, between the structure levels of plastic deformation of irradiated materials, characterized by the known experimental values of athremal stress.

From Eq (4.3) follows at $\left|\left(T-T_{c}\right) / 2 \Delta T_{0}\right|>>1$ that an equality $\sigma_{m}=\sigma_{1,}^{\text {th }}-\sigma_{c}$ is valid if $T<T_{c}$ and the equality $\sigma_{m}=\sigma_{c}-\sigma_{2 \mu}^{t h}$ is fulfilled if $T>T_{c}$ where $\sigma_{1 \mu}^{t h}$ and $\sigma_{2 \mu}^{\text {th }}$ are the theoretical magnitudes of the first (low temperature) and the second (high temperature) athermal plateau, respectively (see Fig.4.1.). This implies that parameter $\sigma_{m}$ is connected with the thermo activated component of irradiated materials stress by $\sigma_{m}=\sigma_{t h}^{*} / 2$.

\subsection{Discussion of model results and experimental data}

The values of empirical parameters $\sigma_{c}, \sigma_{m}, \Delta T_{0}, T_{c}$ are fit by the best coinciding the values of the function (4.3) for corresponding experience temperatures with experimental values of 
the material yield strength. Criterion of fitting the empirical parameter values has been minimization of a quadratic deviation sum of the yield strength experiment values from ones calculated by Eq (4.3) at corresponding experience temperatures for all of the specific materials.

0X18H10T steel samples have been irradiated by $(e, \gamma)$ - beams with energy of $225 \mathrm{MeV}$ up to dose of $10^{25} \mathrm{el} / \mathrm{cm}^{2}$ at temperatures of $170-190^{\circ} \mathrm{C}$. For mechanical experiences, the planar samples of test portion sizes of $10 \times 2 \times 0.3 \mathrm{~mm}$ have been experienced in vacuum at temperatures of $20-1200^{\circ} \mathrm{C}$ with deformation velocity of $0.003 \mathrm{c}^{-1}[15]$. The copper samples of vacuum induced remelting (purity of 99.98) have been irradiated by $\gamma$-beams with energy of $225 \mathrm{MeV}$ up to fluence of $0.1 \mathrm{dpa}$ [15]. The nickel samples have been irradiated by electrons with energy of $225 \mathrm{MeV}$ up to dose of $10^{19} \mathrm{el} / \mathrm{cm}^{2}$ [11]. The 15X2MФA steel samples have been irradiated by neutrons up to fluence of $3 \cdot 10^{20}$ neutron $/ \mathrm{cm}^{2}[11,12]$. The vanadium samples (purity of 99.9) have been irradiated by high energy (of $225 \mathrm{MeV})(e, \gamma)$ - beams up to fluence of 0.01 dpa $[12,15]$. Chromium single crystals have been irradiated by $(e, \gamma)-$ beams up to fluence of $1025 \mathrm{el} / \mathrm{cm}^{2}$ [15]

The summary experimental results for the yield strength temperature dependence of the irradiated materials are shown with Fig.4.2. (fcc lattice materials) and Fig.4.3. (bcc lattice materials) where experimental points are marked by corresponding labels.

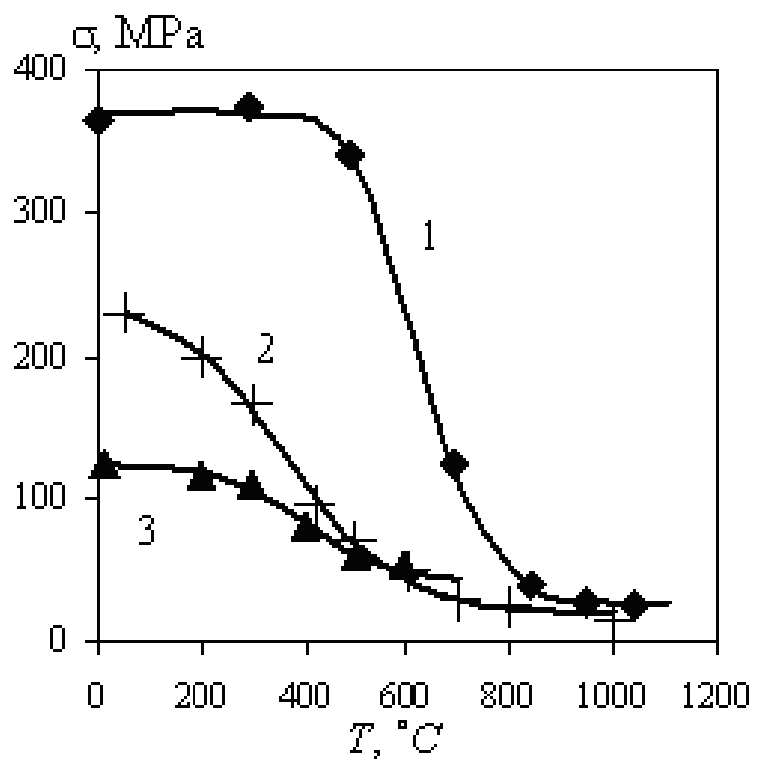

Fig. 4.2. Yield strength temperature dependences of irradiated fcc-materials (point label experiment, solid lines - theoretical plots calculated by Eq (4.3)). 1 - X18H10T steel; 2 nickel; 3 - copper. 


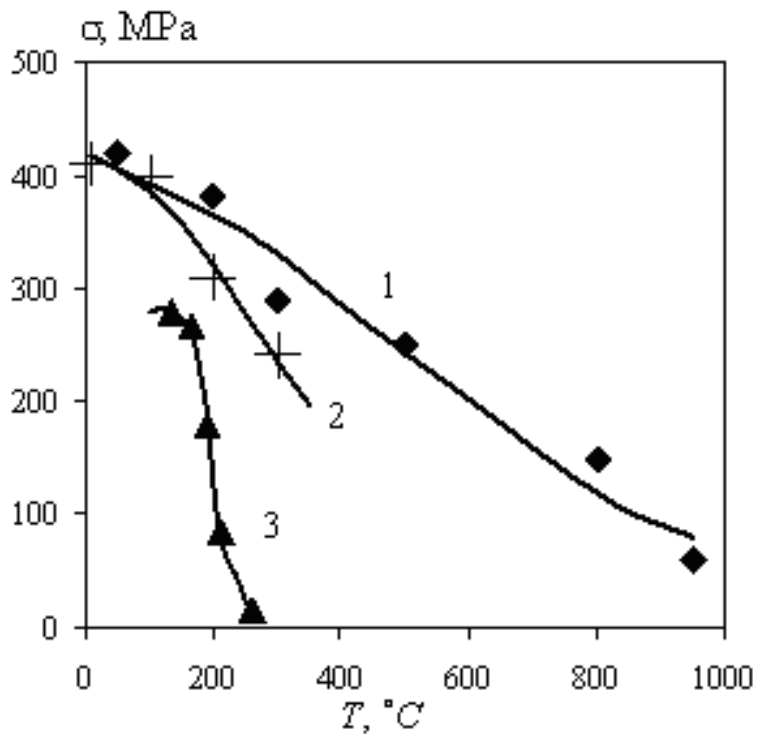

Fig. 4.3. Yield strength temperature dependences of irradiated bcc-materials: 1 - vanadium; 2 - chromium; 3 - 15X2MФA steel.

There are the approximating function (4.3) values of empirical parameters for the different materials, relative error $\varepsilon$ of approximation and confidence quantity $R^{2}$ of approximation (determination coefficient) for all of the dependences in the table. The determination coefficient is close to unit. It means good agreement the proposed theoretical dependences with experimental data for all of the considered materials in the wide experience temperature interval. It should be noted that the relative errors for experimental data to be approximated by the function (4.3) in the case of main fcc-materials (X18H10T steel, copper) are lower than in the case of main bcc-materials (vanadium, chromium).

\begin{tabular}{|l|c|c|c|c|c|c|}
\hline \multicolumn{1}{|c|}{ Material } & $\sigma_{c}, \mathrm{MPa}$ & $\sigma_{m}, \mathrm{MPa}$ & $\Delta T_{0},{ }^{\circ} \mathrm{C}$ & $T_{c},{ }^{\circ} \mathrm{C}$ & $\varepsilon, \%$ & $R^{2}$ \\
\hline 0X18H10T steel & 197.71 & 171.99 & 61.7284 & 634.87 & 1.457 & 0.9997 \\
\hline copper & 87.76 & 35.48 & 58.1395 & 376.70 & 1.799 & 0.9965 \\
\hline nickel & 132.35 & 113.78 & 116.2791 & 357.74 & 4.100 & 0.9972 \\
\hline 15X2MФA steel & 148.78 & 135.35 & 12.9534 & 196.45 & 1.083 & 0.9996 \\
\hline vanadium & 241 & 0.9295 & 243.9024 & 505 & 9.158 & 0.9638 \\
\hline chromium & 277 & 0.5812 & 91.4077 & 251 & 2.848 & 0.9810 \\
\hline
\end{tabular}

Table 1. Empirical parameters of the dependence (4.3).

Also the yield strength temperature dependences of no irradiated materials can be approximated by Eq. (4.3) reasonably enough. For instance, the empirical parameters of no irradiated X18H10T steel are $\sigma_{c}=59.45 \mathrm{MPa}, \sigma_{m}=26.1 \mathrm{MPa}, T_{c}=771.65^{\circ} \mathrm{C}, \varepsilon=4.405 \%$, $R^{2}=0.9814$. Further, it is given the results of comparison of increments for thermo activated $\Delta \sigma^{*}$, and athermal high temperature $\Delta \sigma_{1 \mu}$ and low temperature $\Delta \sigma_{2 \mu}$ of stress components 
obtained experimentally (A) and by a theoretical calculation (B) under radiation up to dose of $10^{25} \mathrm{el} / \mathrm{cm}^{2}$ for $\mathrm{X} 18 \mathrm{H} 10 \mathrm{~T}$ steel.

$\begin{array}{llcc} & & & \\ \Delta \sigma_{1 \mu} \mathrm{MPa} & \ldots \ldots \ldots \ldots \ldots \ldots \ldots & 285 & \mathrm{~B} \\ \Delta \sigma_{2 \mu} \mathrm{MPa} & \ldots \ldots \ldots \ldots \ldots \ldots \ldots \ldots \ldots \ldots \ldots \ldots \ldots & -10 & -7.631 \\ \Delta \sigma^{*} \mathrm{MPa} & \ldots \ldots \ldots \ldots \ldots \ldots \ldots \ldots & 295 & 291.78\end{array}$

According to the data shown at Fig. 4.4., the essential yield strength increment of $\mathrm{X} 18 \mathrm{H} 10 \mathrm{~T}$ steel is observed after radiarion.

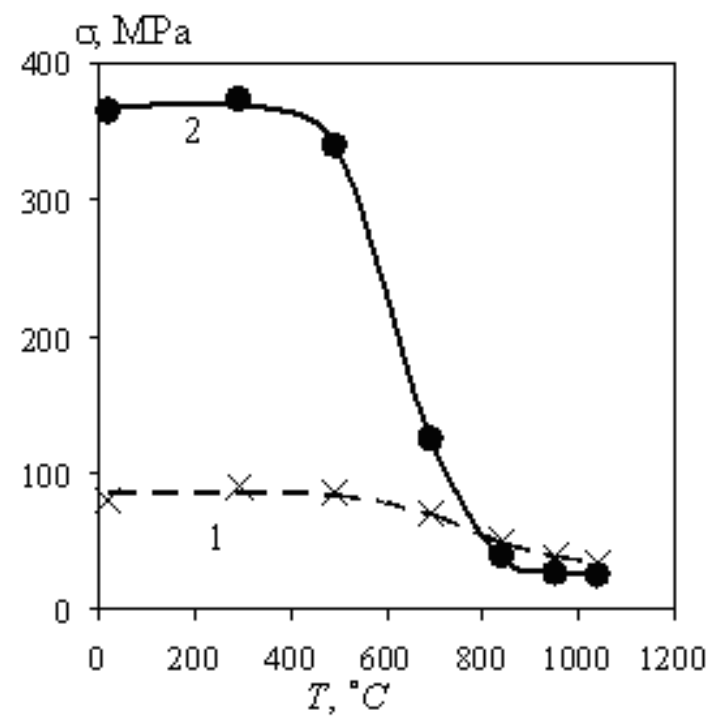

Fig. 4.4. Yield strength temperature dependence of austenic X18H10T steel: 1 - no irradiated, 2 - irradiated by $(e, \gamma)$ - beams up to dose of $10^{25} \mathrm{el} / \mathrm{cm}^{2}$. Point labels are experimental; the lines are plots of theoretical dependence calculated by Eq. (4.3).

\section{Conclusion}

In this chapter, three models are proposed to describe the properties of irradiated deformed polycrystalline materials.

The first model (see section 2) describes the dose dependence of the yield strength of the irradiated material. It is generalization of the model known earlier (see for instance [5]), as the recombination effects of the radiation - induced vacancy and interstitial barriers and their clusterization are taken into account in it. Within the framework of the model formulated, it is found that the saturation quantity of the yield strength decreases with increasing both the intensity of the mutual barrier recombination and the clusterization intensity. It follows to note that in spite of model's assumption for the mean sizes of the radiation-induced barriers $d_{i}$ and the shear modulus $\mu$ to be independent the shear modulus increases practically owing to the radiation point defects come on the dislocation and reduce 
the mean segment length of the dislocation and enhance a degree of the dislocation anchorage. Because of this it can be expected for the obtained dose dependence of the yield strength of the irradiated material to be modified.

The second model describes barrier hardening polycrystalline materials. It is constructed with taking into account interaction of vacancy and interstitial barrier types. In the frame of the proposed model, it can be estimated both contributions to yield strength increment from different type barriers and its total value in dependence on dose.

The third model gives possibility to describe the yield strength dependence of the irradiated materials on experience temperature on a quantitative level. It is based on mechanism of yield strength change as the phase transition between two plastic deformation structure levels characterized by certain values of the athermal stress component. The calculations show radiation promotes the transition of plastic deformation on the higher structure level after that the material undergoes radiation embrittlement. General features found permit to forecast embrittlement temperature intervals of reactor materials in dependence on their mechanical properties.

\section{References}

[1] Tompson M W Defects and Radiation Damage in Metals (London:Cambridge Univ. Press, 1969)

[2] V. Naundorf, "Diffusion in metals and alloys under irradiation," International Journal of Modern Physics B, 6 No. 18 2925--2986 (1992).

[3] A. M. Parshin, I. M. Neklyudov, N.V. Kamyshanchenko, A. N. Tikhonov, et al., @Physics of radiation phenomena and radiation material@ (Publishing BSU, Belgorod, 1998) [in Russian].

[4] N. M. Ghoniem, J. Alhajji and Garner F.A., "Hardening of irradiated alloys due to the simultaneous formation of vacancy and interstitial loops," in @Effect of radiation on materials@ (ASTM, Philadelphia, 1982),pp.1054--1087.

[5] K. Khavanchik, D. Senesh and V. A. Shchegolev, "On saturation of yield strength of copper iiradiated by energy charged particles," PhHOM, No. 2, 5--10 (1989) [in Russian].

[6] ITER Interim Structural Design Criteria (SDC-IC) (ITER Doc.IDoMS G 74 MA 8 01-05-28 W02, 2001).

[7] G.M. Kalinin, B.S. Rodchenkov and V.A. Pechenkin, "Specification of stress limits for irradiated 316L(N)-IG steel in ITER structural design criteria," Journal of Nuclear Materials 329-333, 1615--1618 (2004).

[8] V. C. Neustroyev, Z. E. Ostrovsky, E. V. Boyev and S. V. Belozerov, “Influence of helium accumulation in austenitic steel on evolution of microstructure and radiation damageability of WWER reactor vessel internals materials," in Abstracts of VII Russian conference on reactor material science (NIIAR, Dmitrovgrad, 2007), p 117 [in Russian].

[9] V.V. Krasil'nikov and S.E. Savotchenko, Russian Metallurgy (Metally), Vol. 2009,No. 2, pp. 172-178.

[10] Svetukhin V.V., Sidorenko O.G., Golovanov V.N. and Suslov D.N., Fiz. Khim. Obrab. Mater., 2005, No. 3, pp. 15-20. 
[11] A.M. Parshin, I.M. Neklyudov, N.V. Kamyshanchenko, et al., Physics of Radiation Phenomena and Radiation Material Science (Publishing BSU, Belgorod, 1998) [In Russian].

[12] A. A. Parkhomenko, Microstructure and Radiation Embrittlement of Nickel and OX16P15М3Б Steel, Electron microscopy and Durability of Crystals, No. 9, 103 (1998) [In Russian].

[13] M. P. Zeidlits, L. S. Ozhigov, A. A. Parkhomenko, et al. @Influence High Energy Electron Radiation on Radiation Hardening of Nickel, Vanadium, Iron and Their Alloys, Problems of Atomic Science and Technology. Series: Phys. Rad. Dam., and Rad. Mater. Sc., No. 1 (2), 36 (1975) [In Russian].

[14] V. F. Zelensky, I. M. Neklyudov, L. S. Ozhigov, et al., Utilization of Electron Accelerators for Simulation and Studies of Radiation Effects on Mechanical Properties of Fusion Reactor Materials, J. Nucl. Mater., 207, 280 (1993).

[15] I. M. Neklyudov, V. N. Voevodin, L. S. Ozhigov, et al., Temperature Dependences of Mechanical Properties and Radiation Hardening of Materials, Izv. TulSU. Ser. Physics, No. 4, 87 (2004) [In Russian]

[16] I.M. Neklyudov, I.S. Ozhigov, A.A. Parkhomenko. Utilization of the charge particles of accelerators for simulation of reactor damage effects V.F. Zelensky, // J. Nucl. Mater. - 1993. - Vol. 207. - pp.280-285.

[17] V.F. Zelensky, I.S .Ozhigov, I.M. Neklyudov, Proc. Int. Conf. Irradiation behavior of metallic for fast reactor core components. - France, Corce. 1979. - pp.131-160. 


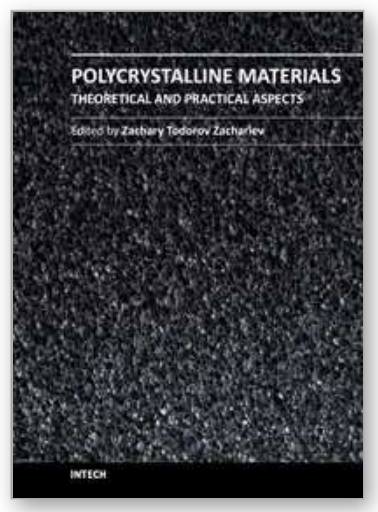

\author{
Polycrystalline Materials - Theoretical and Practical Aspects \\ Edited by Prof. Zaharii Zakhariev
}

ISBN 978-953-307-934-9

Hard cover, 164 pages

Publisher InTech

Published online 20, January, 2012

Published in print edition January, 2012

The book "Polycrystalline Materials - Theoretical and Practical Aspects" is focused on contemporary investigations of plastic deformation, strength and grain-scale approaches, methods of synthesis, structurals, properties, and application of some polycrystalline materials. It is intended for students, post-graduate students, and scientists in the field of polycrystalline materials.

\title{
How to reference
}

In order to correctly reference this scholarly work, feel free to copy and paste the following:

V.V. Krasil'nikov and S.E. Savotchenko (2012). Influence of Irradiation on Mechanical Properties of Materials, Polycrystalline Materials - Theoretical and Practical Aspects, Prof. Zaharii Zakhariev (Ed.), ISBN: 978-953-307934-9, InTech, Available from: http://www.intechopen.com/books/polycrystalline-materials-theoretical-andpractical-aspects/influence-of-irradiation-on-mechanical-properties-of-materials

\section{INTECH}

open science | open minds

\author{
InTech Europe \\ University Campus STeP Ri \\ Slavka Krautzeka 83/A \\ 51000 Rijeka, Croatia \\ Phone: +385 (51) 770447 \\ Fax: +385 (51) 686166 \\ www.intechopen.com
}

\author{
InTech China \\ Unit 405, Office Block, Hotel Equatorial Shanghai \\ No.65, Yan An Road (West), Shanghai, 200040, China \\ 中国上海市延安西路65号上海国际贵都大饭店办公楼 405 单元 \\ Phone: +86-21-62489820 \\ Fax: +86-21-62489821
}


(C) 2012 The Author(s). Licensee IntechOpen. This is an open access article distributed under the terms of the Creative Commons Attribution 3.0 License, which permits unrestricted use, distribution, and reproduction in any medium, provided the original work is properly cited. 